resident choices? http://www.graham-center.org/dam/rgc/documents /publications-reports/monographs-books/Specialty-geography-compressed .pdf. Accessed September 8, 2015.

3. Costa DL, Kahn ME. Power Couples: Changes in the Locational Choice of the College Educated, 1940-1990. Q J Econ. 2000;115(4):1287-1315. doi:10.1162 /003355300555079.

4. Ruggles S, Genadek K, Goeken R, Grover J, Sobek M. Integrated Public Use Microdata Series: Version 6.0. Minneapolis: University of Minnesota; 2015.

5. Health Resources and Services Administration. Shortage designation: health professional shortage areas and medically underserved areas/populations. http://www.hrsa.gov/shortage/index.html. Accessed September 8, 2015

6. Heisler E. Physician Supply and the Affordable Care Act. http://healthcarereform .procon.org/sourcefiles/crs-physician-supply-and-affordable-care-act.pdf. Accessed February 3, 2016.

COMMENT \& RESPONSE

\section{Hip Fracture Surgery vs Elective Total Hip Replacement}

To the Editor The study by Dr Le Manach and colleagues ${ }^{1}$ compared differences in hospital mortality between patients undergoing hip fracture surgery and elective total hip replacement. A population matched for age, sex, and preoperative comorbidities to patients who underwent the 2 operations was created using a multivariable logistic model and a greedy matching algorithm with a 1:1 ratio. This matching model is useful for adjusting the patients' baseline characteristics and controlling selection biases in a retrospective study, but it carries the significant assumption of no unmeasured confounders. That is, all important known factors that can affect measured outcomes must be measured and taken into account in the matching model.

This assumption is unrealistic in this study because many preoperative and intraoperative risk factors that have been independently associated with postoperative shortterm mortality of patients undergoing the 2 operations were not included in the matching model, such as American Society of Anesthesiologists physical status classification, preoperative anemia, hypoproteinemia, cardiac medications, functional status, surgical risk score, intraoperative blood loss, hemodynamic instability and transfusion, and duration of operation. ${ }^{2-5}$ Thus, the work of Le Manach and colleagues cannot provide robust evidence for the increased hospital mortality with hip fracture surgery relative to elective total hip replacement.

Researchers need to perform a large-scale, multicenter, randomized clinical trial in which the innumerable sources of bias that may contaminate the study end points are minimized. If such studies show a consistent beneficial effect of elective total hip replacement relative to hip fracture surgery on postoperative mortality of geriatric patients, the implications for practice are immense.

Fu S. Xue, MD

Gao P. Liu, MD

Rui P. Li, MD

Author Affiliations: Plastic Surgery Hospital, Chinese Academy of Medical Sciences, Beijing, People's Republic of China.
Corresponding Author: Fu Shan Xue, MD, Department of Anesthesiology, Plastic Surgery Hospital, Chinese Academy of Medical Sciences and Peking Union Medical College, 33 Ba-Da-Chu Road, Shi-Jing-Shan District, Beijing 100144, People's Republic of China (xuefushan@aliyun.com).

Conflict of Interest Disclosures: The authors have completed and submitted the ICMJE Form for Disclosure of Potential Conflicts of Interest and none were reported.

1. Le Manach $Y$, Collins $G$, Bhandari $M$, et al. Outcomes after hip fracture surgery compared with elective total hip replacement. JAMA. 2015;314(11):1159-1166.

2. Spahn DR. Anemia and patient blood management in hip and knee surgery: a systematic review of the literature. Anesthesiology. 2010;113(2):482-495.

3. Santaguida PL, Hawker GA, Hudak PL, et al. Patient characteristics affecting the prognosis of total hip and knee joint arthroplasty: a systematic review. Can J Surg. 2008;51(6):428-436.

4. Hooper GJ, Rothwell AG, Hooper NM, Frampton C. The relationship between the American Society Of Anesthesiologists physical rating and outcome following total hip and knee arthroplasty: an analysis of the New Zealand Joint Registry. J Bone Joint Surg Am. 2012;94(12):1065-1070.

5. Jämsen E, Puolakka T, Eskelinen A, et al. Predictors of mortality following primary hip and knee replacement in the aged: a single-center analysis of 1998 primary hip and knee replacements for primary osteoarthritis. Acta Orthop. 2013;84(1):44-53.

To the Editor In an observational study, Dr Le Manach and colleagues ${ }^{1}$ used a 1:1 matching technique and found that the relative risk of in-hospital mortality was higher for patients with hip fracture than those undergoing elective total hip replacement. The authors concluded that this difference could not be adequately explained by patient characteristics and may represent "physiologic mechanisms, such as acute stress and inflammatory states resulting from the fractures." ${ }^{\prime 1}$ However, this conclusion is difficult to justify given the large differences between the elective and trauma populations. Patients undergoing elective total hip replacement are selected for their suitability to undergo a major operation, whereas hip fractures are typically a consequence of physiological deterioration (osteoporosis and falling).

The authors acknowledged the likelihood of residual confounding and selection bias but argued that these cannot explain the large effect size following their extensive efforts to match patients. However, there are many other characteristics that are likely to vary between the groups, including patient factors (eg, frailty and functional capacity) and undiagnosed comorbidities, which are less likely in the elective total hip replacement group that will have undergone comprehensive preoperative assessment.

Most importantly, falls in the elderly are often precipitated by acute illness (eg, urinary tract infection), whereas elective total hip replacement would be postponed under such circumstances. It is not uncommon to admit hip fracture patients with concurrent urinary tract infection, dehydration, acute kidney injury, and uncontrolled atrial fibrillation..$^{2-4}$ In this study, such a patient would have been directly compared with a well counterpart selected and prepared to undergo planned elective surgery. Unfortunately, the authors reported "postoperative outcomes" without clearly distinguishing true postoperative events from acute illness present on admission. The greater risks of "postoperative" stroke, sepsis, and myocardial infarction in the hip fracture group are therefore not surprising. 
The excess relative risk following hip fracture was substantial but unlikely to be explained by a physiological process initiated by injury. Hip fractures are typically lowenergy events and associated with little soft tissue disruption, whereas elective total hip replacement is a major operation that is known to induce a systemic, hypercoagulable state. ${ }^{5}$ It is important to understand the reasons for excess risk among hip fracture patients. However, it is possible that the excess risk can be explained by residual differences between the elective and trauma populations without the need to postulate a new physiological explanation.

\section{David Metcalfe, LLB, MSc, MRCS \\ Daniel C. Perry, PhD, FRCS (Orth)}

Matthew L. Costa, PhD, FRCS (Orth)

Author Affiliations: Center for Surgery and Public Health, Harvard Medical School, Boston, Massachusetts (Metcalfe); Institute of Translational Research, University of Liverpool, Liverpool, United Kingdom (Perry); Nuffield Department of Orthopaedics, Rheumatology and Musculoskeletal Sciences, University of Oxford, Oxford, United Kingdom (Costa).

Corresponding Author: David Metcalfe, LLB, MSc, MRCS, Center for Surgery and Public Health, Harvard Medical School, One Brigham Cir, Boston, MA 02115 (dmetcalfe@bwh.harvard.edu).

Conflict of Interest Disclosures: The authors have completed and submitted the ICMJE Form for Disclosure of Potential Conflicts of Interest. Dr Costa reports receiving grants from SERF, Accueil, X-BOLT Orthopaedics, Orthopaedic Research UK, and the National Institute for Health Research. No other disclosures were reported

1. Le Manach Y, Collins G, Bhandari M, et al. Outcomes after hip fracture surgery compared with elective total hip replacement. JAMA. 2015;314(11):1159-1166.

2. Athar M, O'Loughlin P, Mitra A, Harty J. Characterization of comorbid factors in hip fracture related in-hospital mortality. Ir Med J. 2014;107(9):284-287.

3. Mullen JO, Mullen NL. Hip fracture mortality: a prospective, multifactorial study to predict and minimize death risk. Clin Orthop Relat Res. 1992;(280):214222.

4. Ylinenvaara SI, Elisson O, Berg K, Zdolsek JH, Krook H, Hahn RG. Preoperative urine-specific gravity and the incidence of complications after hip fracture surgery: a prospective, observational study. Eur J Anaesthesiol. 2014:31 (2):85-90.

5. Yang Y, Yao Z, Dai W, Shi P, Luo L, Zhang C. Changes of thrombelastography in patients undergoing elective primary total knee and total hip replacement with low molecular heparin prophylaxis. J Orthop Surg Res. 2014;9:52.

In Reply The potential for unmeasured confounding is a limitation of all observational analyses. Propensity scores can at best achieve balance in measured confounders. ${ }^{1}$ Dr Xue and colleagues correctly assert the importance of including all known factors in the matching model. We were unable to include all covariates that may have an effect on postoperative outcomes (eg, laboratory measurements and chronic treatment prior to surgery), as these were not available in the database. Although frailty and functional status are potentially the most important unmeasured confounders, our sensitivity analysis restricted to patients younger than 60 years who were living at home suggests it is unlikely that this explains the differences in outcomes. This analysis demonstrated an increased risk of mortality (relative risk [RR], 5.80 [95\% CI, 2.9611.35]; $P<$.001) with hip fracture surgery relative to elective total hip replacement.

Dr Metcalfe and colleagues suggest that we have not distinguished postoperative events from acute illness present on admission in those with hip fractures and that this may explain the increased risk of postoperative complications. We only included patients who underwent surgery and as such the primary outcome (ie, in-hospital mortality) was always a postoperative event. Although diagnostic coding did not allow us to distinguish whether secondary outcomes happened before or after fracture surgery, we excluded patients who had surgery beyond 7 days after admission for a hip fracture. Given that it is uncommon for patients who have an acute event like a stroke, myocardial infarction, or sepsis to undergo surgery within 7 days makes it unlikely that these events occurred prior to hip fracture surgery.

We agree that a randomized clinical trial (RCT) is the preferable approach to demonstrate a causal relationship between a potential cause (eg, preoperative treatment) and an effect (eg, postoperative outcome). However, elective total hip replacement and hip fracture surgery cannot be compared in an RCT for ethical reasons (ie, patients cannot be randomized to have a hip fracture and then surgery). Only potentially modifiable factors can be tested in an RCT. We are conducting a large international RCT of accelerated surgery within 6 hours of a hip fracture diagnosis vs standard care in which surgery typically occurs within 20 to 48 hours after a hip fracture is diagnosed. ${ }^{2}$

Metcalfe and colleagues also point out that hip fractures are low-energy events and associated with little soft tissue disruption. However, blood loss associated with hip fracture is frequently larger than that observed with total hip replacement surgery. ${ }^{3,4}$ They suggest that there is no need to postulate a new physiological mechanism to explain the inferior outcomes for patients with a hip fracture relative to patients undergoing an elective total hip replacement. We disagree. We believe there is a need to explore new ideas that are potentially amenable to interventions that may allow improvements in the poor outcomes associated with hip fractures. To the extent that the physiological processes initiated by a hip fracture play a role in the poor outcomes, minimizing the time patients are exposed to these physiological processes may improve outcomes. Large RCTs are needed to resolve this issue.

\section{Yannick Le Manach, MD, PhD}

Gary S. Collins, $\mathrm{PhD}$

\section{P. J. Devereaux, MD, PhD}

Author Affiliations: Population Health Research Institute, David Braley Cardiac, Vascular and Stroke Research Institute, Hamilton, Ontario, Canada (Le Manach, Devereaux); Centre for Statistics in Medicine, University of Oxford, Oxford, United Kingdom (Collins).

Corresponding Author: Yannick Le Manach, MD, PhD, Population Health Research Institute, David Braley Cardiac, Vascular and Stroke Research Institute, Perioperative Medicine and Surgical Research Institute, 237 Barton St E, Hamilton, ON L8L 2X2, Canada (yannick.lemanach@phri.ca).

Conflict of Interest Disclosures: The authors have completed and submitted the ICMJE Form for Disclosure of Potential Conflicts of Interest. Dr Devereaux reports receiving grant funding from Abbott Diagnostics, Boehringer Ingelheim, Covidien, Octopharma Plasma, Roche Diagnostics, and Stryker. No other disclosures were reported

1. Austin PC. An introduction to propensity score methods for reducing the effects of confounding in observational studies. Multivariate Behav Res. 2011;46 (3):399-424. 
2. Hip Fracture Accelerated Surgical Treatment and Care Track (HIP ATTACK) Investigators. Accelerated care vs standard care among patients with hip fracture: the HIP ATTACK pilot trial. CMAJ. 2014;186(1):E52-E60.

3. Foss NB, Kehlet $\mathrm{H}$. Hidden blood loss after surgery for hip fracture. J Bone Joint Surg Br. 2006;88(8):1053-1059.

4. Smith GH, Tsang J, Molyneux SG, White TO. The hidden blood loss after hip fracture. Injury. 2011;42(2):133-135.

\section{Implementing Quality Improvement for Psychosocial Interventions}

To the Editor A Viewpoint by Drs Pincus and England summarized a new report by the Institute of Medicine on improving the quality of psychosocial interventions for mental and substance use disorders. ${ }^{1}$ We wish to draw attention to advances in intervention science that could facilitate implementation of recommendations in the report.

The Behavior Change Technique Taxonomy addresses the recommendation for a common terminology for specifying elements of psychosocial intervention elements. ${ }^{2}$ This taxonomy was developed using consensus methods and includes a hierarchically structured catalog of 93 distinct intervention elements. Use of this taxonomy would improve the clarity and synthesis of research. Widespread adoption could further the goals of identifying key elements within and across interventions, discovering optimal dosing and sequencing of elements, and connecting elements to purported moderators or mechanisms.

New methods for research synthesis can facilitate implementation of the recommendations regarding high-quality systematic reviews of psychosocial intervention elements. ${ }^{3}$ Theory-based meta-regression involves using theoretical frameworks to select intervention elements for metaanalytic investigation. Individual participant-level data metaanalysis is much more powerful than meta-analysis using aggregate data for identifying mediators and moderators of intervention effects. Network meta-analysis can use all relevant trials in one analysis to probabilistically rank multiple interventions according to their comparative effectiveness. Qualitative meta-syntheses offer an explicit and transparent method for developing robust descriptive and analytic themes about intervention implementation across a body of literature. Greater use of these research synthesis techniques can inform clinical guidelines and implementation tools on psychosocial intervention elements.

The report recommends developing performance measures to guide continuous quality improvement for psychosocial interventions. As it notes, standardization is limited because consensus is often lacking about which outcomes and measures should be prioritized. The Core Outcome Measures in Effectiveness Trials Initiative aims to further the development of "core outcome sets"-based on research evidence and stakeholder consensus-that specify the most important outcomes to measure when evaluating interventions for a specific condition and the best extant tools to measure these outcomes. ${ }^{4}$ Although originally intended for use in clinical trials, core outcome sets are increasingly used to select outcomes for clinical audit and performance measurement-and could be developed for psychosocial interventions targeting mental health and substance use disorders for this purpose.

Such advances may be useful to implementation of the report's recommendations and may facilitate the goal of improving scientific evidence and quality improvement for psychosocial interventions.

\section{Sean Grant, DPhil \\ Evan Mayo-Wilson, DPhil \\ Paul Montgomery, DPhil}

Author Affiliations: RAND Corporation, Santa Monica, California (Grant); Center for Clinical Trials and Evidence Synthesis, Johns Hopkins Bloomberg School of Public Health, Baltimore, Maryland (Mayo-Wilson); Centre for Evidence-Based Intervention, University of Oxford, Oxford, United Kingdom (Montgomery).

Corresponding Author: Sean Grant, DPhil, RAND Corporation, 1776 Main St, PO Box 2138, Santa Monica, CA 90407-2138 (sgrant@rand.org).

Conflict of Interest Disclosures: The authors have completed and submitted the ICMJE Form for Disclosure of Potential Conflicts of Interest and reported being investigators on the Consolidated Standards of Reporting Trials for Social and Psychological Interventions (CONSORT-SPI).

1. Pincus HA, England MJ. Improving the quality of psychosocial interventions for mental and substance use disorders: a report from the IOM. JAMA. 2015;314 (12):1227-1228.

2. Michie $S$, Richardson $M$, Johnston $M$, et al. The behavior change technique taxonomy ( $v 1$ ) of 93 hierarchically clustered techniques: building an international consensus for the reporting of behavior change interventions. Ann Behav Med. 2013;46(1):81-95.

3. Melendez-Torres GJ, Bonell C, Thomas J. Emergent approaches to the meta-analysis of multiple heterogeneous complex interventions. BMC Med Res Methodol. 2015;15:47.

4. Williamson $P$, Altman D, Blazeby J, Clarke M, Gargon E. Driving up the quality and relevance of research through the use of agreed core outcomes. $J$ Health Serv Res Policy. 2012;17(1):1-2.

In Reply We agree with Dr Grant and colleagues that the advances in implementation science they note have the potential to make an important contribution toward implementation of the recommendations of the Institute of Medicine to improve the quality of psychosocial interventions for mental and substance use disorders. In particular, their comments support the recommendation for "developing a common terminology for describing and classifying the elements of evidencebased psychosocial interventions," as well as recommendations related to evidence synthesis and future research needs. Furthermore, we believe that the Behavioral Change Technique Taxonomy is a useful effort toward developing an ontology of intervention elements that would not only be useful for research on psychosocial interventions but also lay the groundwork for advancing needed quality measurement and improvement strategies for mental illness and substance abuse care.

\section{Harold Alan Pincus, MD Mary Jane England, MD}

Author Affiliations: Columbia University, New York-Presbyterian Hospital, New York, New York (Pincus); School of Public Health, Boston University, Boston, Massachusetts (England).

Corresponding Author: Harold Alan Pincus, MD, Columbia University Medical Center, New York State Psychiatry Institute, 1051 Riverside Dr, Unit 09. New York, NY 10032 (pincush@nyspi.columbia.edu). 